\title{
Seguimento ambulatorial de um grupo de prematuros e a prevalência do aleitamento na alta hospitalar e ao sexto mês de vida: contribuições da Fonoaudiologia
}

\author{
Outpatient follow-up of a group of premature infants and the \\ prevalence of breastfeeding at discharge and at six months: \\ Speech-Language Pathology contributions
}

\author{
Aliana Eduarda Czechowski ${ }^{1}$, Cristina Ide Fujinaga ${ }^{2}$
}

\begin{abstract}
RESUMO
Objetivo: Verificar a prevalência do aleitamento materno em prematuros com peso acima de $1500 \mathrm{~g}$, na alta hospitalar e até o $6^{\circ}$ mês de vida. Métodos: Trata-se de um estudo descritivo observacional, retrospectivo, de caráter quantitativo, baseado na análise de prontuários de bebês nascidos entre janeiro de 2006 e dezembro de 2007, que foram assistidos pelo serviço de Fonoaudiologia na unidade pediátrica e ambulatório de Fonoaudiologia de um Hospital de Base. Foram incluídos recém-nascidos pré-termo de baixo peso, com ausência de doenças congênitas cardíacas, pulmonares ou síndromes genéticas e índice de APGAR maior que três no $1^{\circ}$ minuto e cinco no $5^{\circ}$ minuto. Resultados: Dos 156 prontuários estudados, constatou-se que a prevalência de aleitamento materno exclusivo na alta hospitalar foi de 58,3\%. Aos seis meses de vida do bebê foi observado uma prevalência de $22,2 \%$ em aleitamento materno exclusivo. Conclusão: A prevalência do aleitamento materno exclusivo mostrou-se bastante aquém do valor recomendado pela Organização Mundial de Saúde, que é de aleitamento materno exclusivo até o sexto mês de vida.
\end{abstract}

Descritores: Recém-nascido; Peso ao nascer; Prematuro; Aleitamento materno; Desmame

\section{INTRODUÇÃO}

Vem crescendo no mundo, inclusive no Brasil, o incentivo e a valorização da prática do aleitamento materno (AM). Especialmente em casos de prematuridade e baixo peso ao nascer, a amamentação ainda é um desafio para os profissionais envolvidos, mães e bebês pré-termo. Com frequência, observa-se o desmame precoce, sendo de grande importância o envolvimento de profissionais preparados para oferecer suporte e manejo clínico hospitalar, almejando o sucesso do aleitamento materno ${ }^{(1)}$.

Trabalho realizado no Hospital de Base da Fundação Faculdade de Medicina de São José do Rio Preto - FUNFARME/FAMERP - São José do Rio Preto (SP), Brasil, com bolsa concedida pela Fundação do Desenvolvimento Administrativo, vinculado ao Programa de Aprimoramento Profissional do Estado de São Paulo.

(1) Pós-graduanda (Especialização) em Motricidade Orofacial: enfoque em disfagia e atuação em âmbito hospitalar, da Universidade Tuiuti do Paraná UTP - Curitiba (PR), Brasil; Fonoaudióloga do Hospital Regional do Sudoeste - Francisco Beltrão (PR), Brasil.

(2) Pós-doutoranda, Professora Adjunto B do Departamento de Fonoaudiologia da Universidade Estadual do Centro-Oeste - UNICENTRO - Irati (PR), Brasil. Endereço para correspondência: Aliana Eduarda Czechowski. R. Mário de Barros, 1700, Nossa Senhora de Lourdes, Dois Vizinhos (PR), Brasil, CEP: 85660-000. E-mail: alianaeduarda@yahoo.com.br

Recebido em: 7/7/2009; Aceito em: 28/4/2010
De fato, a amamentação constitui o modo mais natural e seguro para a alimentação do lactente, ideal para o seu crescimento e desenvolvimento. Para tanto, a Organização Mundial da Saúde (OMS) preconiza o aleitamento materno exclusivo até o $6^{\circ}$ mês de vida do bebê, podendo ser mantido beneficamente até os dois anos de idade, com complementação alimentar ${ }^{(2)}$.

A preocupação com a qualidade de vida dos prematuros aumenta o incentivo à amamentação e essa incitação justificase por diversas razões. Além de influenciar na saúde biológica e emocional da díade mãe-filho, o leite materno apresenta propriedades imunológicas e nutritivas, não tem custos financeiros, e oferece melhor proteção a antioxidantes que os leites artificiais, diminuindo a ocorrência de enterocolite necrosante, displasia broncopulmonar, hemorragia intraventricular e retinopatia da prematuridade ${ }^{(1,3)}$.

As pesquisas apontam que prematuros e de baixo peso, amamentados no peito da mãe, apresentam menor tempo de internação hospitalar, melhor prognóstico para o desenvolvimento neurológico, diminuição da perda de peso, diminuição do índice de doenças crônicas e agudas e aumento de sobrevida, em relação àqueles amamentados com leite industrializado ${ }^{(4-7)}$.

Além dos benefícios nutricionais, imunológicos, emocio- 
nais e socioeconômicos, a amamentação natural contribui para a saúde fonoaudiológica, pois está relacionada ao crescimento e desenvolvimento craniofacial e motor-oral do recém-nascido (RN). Assim, previne alterações dos órgãos fonoarticulatórios e das funções neurovegetativas, tais como respiração, mastigação, deglutição e articulação dos sons da fala ${ }^{(8)}$.

Dessa forma, faz-se de extrema importância a valorização, incentivo e apoio à prática da amamentação, tanto para a qualidade de vida, quanto para o vínculo mãe-bebê, auxiliando o processo de aproximação e aceitação do nascimento de um filho prematuro e/ou de baixo peso, diferente do filho idealizado pela mãe. Assim, o AM pode ser uma maneira positiva e factível de estabelecer uma ligação afetiva entre mãe e filho(9,10).

O fonoaudiólogo pode exercer papel decisivo no sucesso do aleitamento materno, incluindo estratégias de incentivo e apoio, além de envolver diretamente a mãe nos cuidados à alimentação do prematuro.

A elaboração dessa pesquisa justifica-se pela escassez de estudos referentes à prevalência do aleitamento materno na população de prematuros e de baixo peso ao nascer, principalmente no seguimento após a alta hospitalar. Apesar da recomendação da OMS de aleitamento materno exclusivo até o $6^{\circ}$ mês, muitas vezes o desmame ocorre precocemente; portanto, torna-se indispensável verificar como se dá a amamentação após a alta hospitalar. Nesse sentido, o presente estudo teve como objetivo investigar a prevalência do aleitamento materno em prematuros nascidos com peso acima de 1500 gramas, por ocasião da alta hospitalar, até o sexto mês de vida, durante o segmento ambulatorial.

\section{MÉTODOS}

Foi realizada uma análise retrospectiva de prontuários de prematuros assistidos na unidade de pediatria e ambulatório de um Hospital de Base, durante o período neonatal, até o $6^{\circ}$ mês de vida do bebê. A população estudada foi selecionada pelo método de amostragem não-probabilístico acidental ou por conveniência. O estudo é do tipo descritivo observacional, retrospectivo, de caráter quantitativo ${ }^{(11)}$.

Deste segmento populacional, foram selecionados prontuários de prematuros nascidos no período de janeiro de 2006 a dezembro de 2007 , respeitando-se os seguintes critérios de inclusão: recém nascidos com idade gestacional inferior a 37 semanas, determinada pelo método Capurro Somático e registrada pelo médico ao nascimento; ausência de doenças congênitas cardíacas ou pulmonares severas e síndromes genéticas associadas à prematuridade; índice de APGAR maior que três ou cinco no $1^{\circ}$ e $5^{\circ}$ minuto, respectivamente. No que se refere às condições maternas, foram considerados os aspectos: ausência de doenças ou procedimentos terapêuticos que contra-indicassem ou pudessem interferir no aleitamento materno; aceitação e desejo em amamentar.

Todos os bebês atendidos pelo serviço de Fonoaudiologia durante o período de internação hospitalar recebiam orientações quanto ao manejo da amamentação e sua importância, e por ocasião da alta hospitalar agendava-se a primeira consulta fonoaudiológica para seguimento ambulatorial. $\mathrm{O}$ atendimento ambulatorial ocorre mensalmente até $\mathrm{o} 1^{\circ}$ ano de vida do bebê.
Após esta idade, a frequência das consultas é determinada em cada caso, conforme as necessidades do bebê.

As consultas realizadas neste setor têm como objetivo acompanhar o desenvolvimento do bebê quanto à alimentação, audição, linguagem e motricidade orofacial. Para tanto, fazse anamnese com a mãe, observação e avaliação da mamada, screening auditivo e orientações pertinentes. Os dados são registrados em prontuário e tais informações formam a base de dados do presente estudo.

Assim, para a seleção da amostra, foi utilizado o livro de registro de atendimento hospitalar da Fonoaudiologia. Em seguida, foram anotados os dados coletados em prontuário. Participaram desta pesquisa 156 recém-nascidos pré-termos de baixo peso atendidos pelo serviço de Fonoaudiologia durante a internação hospitalar e 36 que compareceram ao atendimento ambulatorial aos seis meses de idade. Desta forma a amostra foi composta, na alta hospitalar, por 156 prematuros e aos seis meses, por 36 prematuros.

Considerou-se, tanto no momento da alta hospitalar quanto nas consultas ambulatoriais no segmento com a Fonoaudiologia, três categorias de aleitamento materno: aleitamento materno exclusivo, quando registrado aleitamento em peito materno e ausência de complementação da mamada; aleitamento misto, quando registrado aleitamento em peito materno, mais complementação da mamada, com uso do copo ou mamadeira; e aleitamento artificial, quando não há registro de aleitamento em peito, com uso de mamadeira.

As variáveis relacionadas à mãe e ao $\mathrm{RN}$ tais como, tipo de parto, escolaridade materna, número de gestações anteriores, desconforto respiratório, gemelaridade, relação peso/idade gestacional e gênero foram relacionadas estatisticamente à presença/ausência de aleitamento materno exclusivo na alta hospitalar.

Os dados foram tabulados, submetidos à análise estatística e apresentados em forma de gráficos e tabelas. Para testar o intervalo de confiança de uma proporção foi utilizado o teste de Mood para mediana, baseado no Qui-quadrado de Pearson. Para as categorias com células de baixa frequência, foi utilizado o teste Fisher.

Esta pesquisa foi aprovada pelo Comitê de Ética e Pesquisa da Faculdade de Medicina de São José do Rio Preto, cujo processo é o número 4368/2008, e autorizada pelo Serviço de Arquivamento Médico e Estatística (SAME) do Hospital de Base da Fundação Faculdade de Medicina de São José do Rio Preto. Em respeito aos princípios éticos, foi mantido o anonimato dos prontuários, conforme a resolução 196/96, com o propósito de assegurar a proteção dos direitos humanos.

\section{RESULTADOS}

Dos 156 prontuários analisados, 67 (43\%) eram do gênero feminino e 89 (57\%) do gênero masculino. A gemelaridade foi encontrada em 22 prontuários (14\%). O parto realizado por meio da cesárea representou $73,7 \%$ do total. Em relação à idade materna, 60,9\% tinham entre 15 e 24 anos. As mães, majoritariamente $(42,9 \%)$, não haviam concluído o primeiro grau do ensino fundamental. Quanto à idade gestacional, registrada pelo médico ao nascimento, 64 (41\%) dos prematuros possuía idade igual ou superior a 34 semanas, seguido de 62 
Tabela 1. Estimação de proporção para as variáveis relacionadas à mãe e ao recém-nascido e sua associação com o aleitamento materno exclusivo na alta

\begin{tabular}{|c|c|c|c|}
\hline Categoria de variável & & Frequência de AME (\%) & Valor de $p$ \\
\hline \multirow{2}{*}{ Tipo de parto } & Normal & 22,73 & \multirow{2}{*}{0,745} \\
\hline & Cesárea & 77,27 & \\
\hline \multirow{2}{*}{ Escolaridade } & Até $1^{\circ}$ grau incompleto & 61,36 & \multirow{2}{*}{0,354} \\
\hline & $1^{\circ}$ grau completo ou mais & 38,64 & \\
\hline \multirow{2}{*}{$\mathrm{N}^{\circ}$ de gestações anteriores } & Nenhuma & 40,00 & \multirow{2}{*}{0,010} \\
\hline & Uma ou mais & 60,00 & \\
\hline Gemelaridade & & 25,81 & $0,004^{*}$ \\
\hline Presença de desconforto respiratório & & 72,53 & 0,665 \\
\hline \multirow{2}{*}{ Relação peso/idade gestacional } & $A I G$ & 98,89 & \multirow{2}{*}{0,065} \\
\hline & PIG & 1,11 & \\
\hline \multirow{2}{*}{ Gênero } & $\mathrm{F}$ & 46,15 & \multirow{2}{*}{0,632} \\
\hline & M & 53,85 & \\
\hline
\end{tabular}

Teste da Mediana de Mood e Teste de Fischer

* Valores significativos $(p \leq 0,01)$

Legenda: $\mathrm{AME}=$ aleitamento materno exclusivo; $\mathrm{AIG}=$ adequado para a idade gestacional; $\mathrm{PIG}=$ pequeno para a idade gestacional; $\mathrm{F}=$ feminino; $\mathrm{M}=\mathrm{masculino}$

$(39,8 \%)$ com idade entre 32 e 34 semanas. No que se refere ao peso, $97(62 \%)$ dos prematuros pesavam entre 1500 e $2000 \mathrm{~g}$ e $59(38 \%)$ pesavam entre 2001 e 2500 g. Cento e cinquenta e um bebês $(96,8 \%)$ eram adequados para a idade gestacional, ao passo que apenas cinco bebês $(3,2 \%)$ eram pequenos para a idade gestacional. $\mathrm{O}$ desconforto respiratório foi registrado em 114 prontuários, ou seja, em 73,1\% da amostra.

De acordo com os tipos de prematuridade (limítrofe, moderada e extrema), verificou-se que $34(21,8 \%)$ dos recémnascidos apresentavam idade gestacional entre 35 e 36 semanas (limítrofe), 118 (75,7\%) dos recém nascidos entre 31 e 34 semanas (moderada) e quatro bebês $(2,5 \%)$ abaixo ou igual a 30 semanas de gestação (extrema). A prematuridade moderada representou a maioria $(75,7 \%)$ e a prematuridade extrema foi observada em apenas quatro casos $(2,5 \%)$.

Sobre as variáveis relacionadas à mãe e ao RN, a Tabela 1 mostra que existe associação entre a prevalência do aleitamento materno e a gemelaridade, com evidência da minoria estar em aleitamento materno exclusivo.

$\mathrm{Na}$ alta hospitalar foi constatada prevalência de aleitamento materno exclusivo de $58,3 \%$, ou seja, dos 156 recém-nascidos, 91 estavam sendo amamentados exclusivamente no peito materno, observando-se taxas de aleitamento materno misto e artificial, respectivamente em $21,8 \%$ e $19,9 \%$ dos neonatos, como mostra a Figura 1. A partir desses resultados, o teste da mediana de Mood indicou que há evidência de que a maioria está em aleitamento materno exclusivo.

No que se refere ao seguimento ambulatorial, dos 156 prontuários estudados, verificou-se que apenas 36 bebês foram atendidos pelo serviço de Fonoaudiologia aos seis meses de idade e a prevalência do aleitamento materno exclusivo foi de $22,2 \%$, contra $77,8 \%$ em ausência de aleitamento materno exclusivo (33,3\% em aleitamento misto e $44,5 \%$ em aleitamento artificial).

Os resultados da Figura 2 evidenciam o declínio das taxas de aleitamento materno após a alta hospitalar. Em relação ao $\mathrm{N}$ amostral, no segmento ambulatorial observa-se o grande

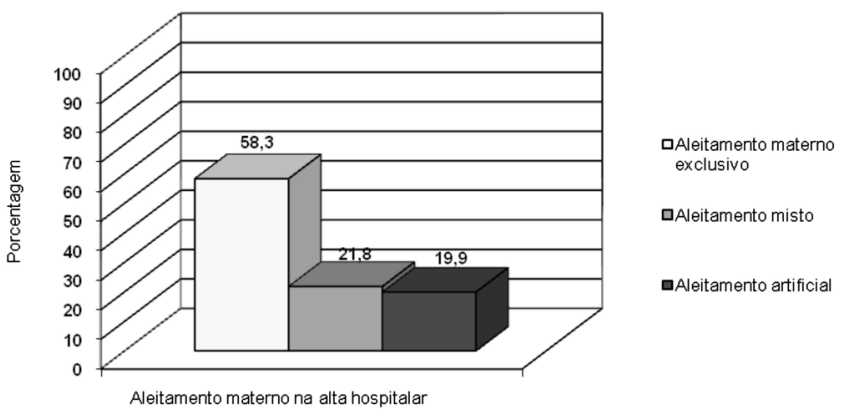

Figura 1. Incidência do aleitamento na alta hospitalar

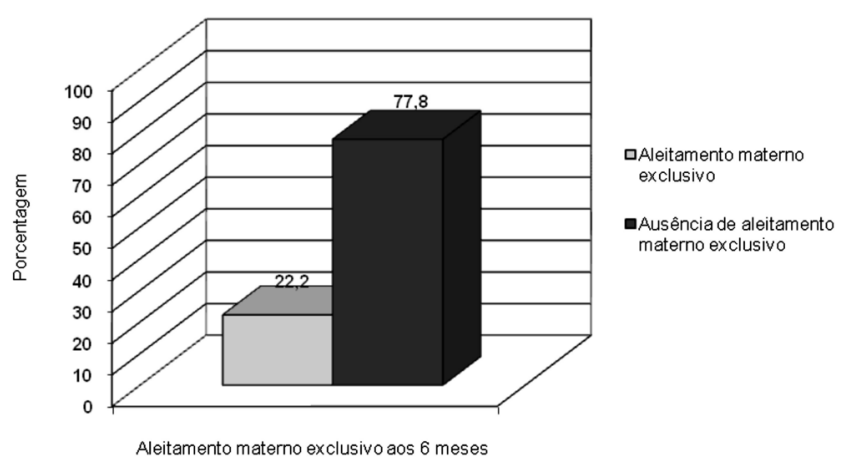

Figura 2. Prevalência do aleitamento materno exclusivo aos seis meses

número de faltas das mães aos atendimentos fonoaudiológicos.

Na Figura 3, quanto à gemelaridade, o fator 1 explica a dicotomia exclusivo versus não exclusivo, com $91,8 \%$ da dependência total; o fator 2 , com explicação de $7,8 \%$ da dependência total, contrapõe aleitamento misto a artificial, associando ao misto gemelar 1, e ao artificial gemelar 2.

As variáveis tipo de parto, escolaridade materna, número de gestações anteriores, desconforto respiratório, relação peso/ idade gestacional e gênero não foram associadas ao aleitamento materno exclusivo (AME) na alta hospitalar. 


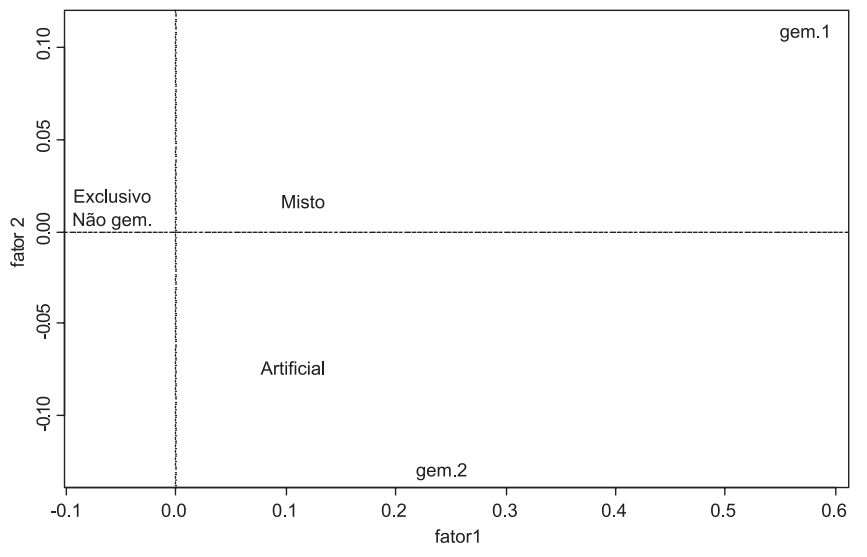

Figura 3. Dicotomia exclusivo x não exclusivo em relação à gemelaridade

\section{DISCUSSÃO}

Poucos são os estudos que verificaram a prevalência do AME em prematuros e recém-nascidos de baixo-peso. Em Belo Horizonte (MG), foi realizado um estudo cujo objetivo foi avaliar as taxas e o padrão de AME dos recém-nascidos internados em berçário de alto risco no momento de sua alta hospitalar e analisar os potenciais fatores que interferem na prática do AME. Os autores observaram prevalência de $36 \%$ de AME na alta antes da Iniciativa Hospital Amigo da Criança (IHAC) e 54,7\% após a implantação da IHAC. Quanto às variáveis relacionadas ao recém-nascido e sua associação com o tipo de aleitamento na alta, especificadamente, em relação ao peso de nascimento e à idade gestacional, o estudo constatou prevalência de AME de 38,5\% em recém-nascidos com peso inferior a 2500 g e 39,2\% em recém-nascidos com idade gestacional inferior a 37 semanas. Porém, não houve associação significativa e os dados não foram relacionados com o período anterior e posterior à implantação da $\mathrm{IHAC}^{(12)}$.

Os fatores de risco associados para o não aleitamento materno foram tempo de dieta por sonda gástrica, número pequeno de consultas pré-natais, uso da translactação, peso ao nascer menor do que $2500 \mathrm{~g}$ e implementação da IHAC ${ }^{(12)}$. No presente estudo, tais variáveis não foram analisadas, apenas a gemelaridade foi associada ao não aleitamento materno. Há uma lacuna na literatura sobre aleitamento materno em gêmeos prematuros. Entretanto, a experiência clínica indica que as mães de gemelares possuem maior dificuldade em manter o aleitamento materno exclusivo devido ao cansaço e necessidade de complementação da alimentação.

$\mathrm{Na}$ instituição estudada, o complemento do aleitamento materno é oferecido no copo ou mamadeira. O copo é apontado como um método alternativo seguro para alimentação do prematuro até que o mesmo seja capaz de se alimentar por via oral ${ }^{(13,14)}$. Entretanto, a literatura aponta outros métodos alternativos para complementação do aleitamento, que são mais fisiológicos, envolvem diretamente a mãe no cuidado e promovem a maior prevalência de aleitamento materno na alta hospitalar. Os métodos recomendados são a relactação, translactação e sonda-peito ${ }^{(15)}$. Outra vantagem de tais métodos é que neles a mãe possui papel ativo e sua lactação é estimulada, aumentando sua produção láctea e confiança no cuidado ao seu filho ${ }^{(12,15)}$.

Outro estudo, realizado em uma instituição com o título de Hospital Amigo da Criança, encontrou índices, respectivamente, de $28,4 \%, 48,3 \%$ e $23,2 \%$ de aleitamento materno exclusivo, misto e artificial em prematuros. Ocorreu associação entre a prevalência do aleitamento materno com o peso e a idade gestacional ao nascimento ${ }^{(16)}$.

A atual pesquisa revelou o uso de mamadeira como o método de alimentação artificial com os prematuros. Alguns autores mencionam como a principal causa do desmame precoce, a introdução da mamadeira para alimentação infantil ${ }^{(17,18)}$. Ressalta-se que a sucção no seio materno, comparada à sucção realizada com o bico da mamadeira, não segue os mesmos princípios e que a introdução de bicos, sejam de mamadeira ou chupeta, promove a confusão de bicos, dificultando o aleitamento materno ${ }^{(8)}$.

Estudos mostram que a alimentação artificial pode exercer efeito deletério sobre o aleitamento materno, independente do método utilizado (copo ou mamadeira). Além disso, entre as crianças expostas à chupeta, no período neo e pós-natal, observa-se menor incidência de aleitamento materno exclusivo ${ }^{(14,18)}$.

A literatura ainda é escassa no que tange à prevalência do aleitamento materno em prematuros após a alta hospitalar. Estudo realizado com 14 recém nascidos pré-termo, nascidos no Hospital do Servidor Público Estadual, verificou que 1 mês após a alta, 28,5\% dos prematuros estavam em aleitamento materno exclusivo ${ }^{(19)}$. Outro estudo realizado em Maringá (PR) constatou prevalência de $34,3 \%$ de aleitamento materno exclusivo em recém-nascidos com peso igual ou inferior a $2500 \mathrm{~g}$ e $56,3 \%$ em RNs pré-termo aos quatro meses de idade ${ }^{(20)}$.

Pesquisa realizada em Campinas (SP), na qual foram analisados 165 prontuários de prematuros de muito baixo peso, observou que a frequência do aleitamento materno no primeiro ano de vida foi maior no grupo que utilizou o copo durante a transição alimentar (67,2\% aos três meses, 41,0\% aos seis meses e $33,2 \%$ aos 12 meses) $)^{(21)}$.

Salienta-se que a redução da prevalência do aleitamento materno até o $6^{\circ}$ mês pode estar relacionada também com a desistência da mãe do seguimento ambulatorial fonoaudiológico. Apesar de existirem, atualmente, programas de suporte à puérpera durante a gestação, que enfatizam a importância do aleitamento materno, há muito mais para ser feito do que simplesmente "informar". É imprescindível a atenção e a preocupação não apenas com a mãe "nutriz", mas com a mãe "mulher", aquela que precisa ter alguma razão para sustentar um atendimento fonoaudiológico.

Chama a atenção o fato da prática do aleitamento materno estar vinculada, inclusive, a uma questão social, na qual, a mãe retorna ao trabalho; e é nesse contexto que frequentemente, a alimentação artificial é introduzida. Nesse sentido, entendese porque os índices de aleitamento materno exclusivo caem, evidenciando uma minoria em amamentação exclusiva no peito. Apesar desta instituição, na qual o estudo foi realizado, oferecer serviço fonoaudiológico no seguimento ambulatorial, contatou-se que a minoria dos recém-nascidos assistidos durante a internação compareceu a este atendimento e, concomitantemente, observou-se o declínio das taxas de aleitamento 
materno exclusivo, mostrando índices aquém do recomendado pelo Ministério da Saúde. Dados semelhantes foram encontrados em estudo realizado no Nordeste brasileiro ${ }^{(17)}$, e no município de Passo Fundo (RS) ${ }^{(22)}$.

Em uma pesquisa de revisão de literatura foram elencados os principais determinantes para o desmame precoce, tais como o conhecimento das mães para o manejo da amamentação natural, a primiparidade, o uso da chupeta e o nascimento do bebê em hospital não credenciado à IHAC ${ }^{(23)}$. A variável trabalho materno não foi considerada relevante. Porém, um estudo transversal realizado com mães de crianças menores de seis meses de idade, matriculadas em programa de puericultura, na cidade de Ribeirão Preto (SP), mostrou que os principais motivos para a introdução da mamadeira, segundo a opinião materna, foi a diminuição do leite, seguido do trabalho exter$\mathrm{no}^{(24)}$. No que tange a este último, outro estudo mostrou que as mães que trabalhavam fora de casa amamentaram por um período de tempo maior, considerando que esta variável foi associada à maior escolaridade ${ }^{(25)}$.

Tais associações não foram verificadas no presente estudo, que não se propôs a investigar as possíveis causas para o desmame precoce. Contudo, é imprescindível a realização de outras pesquisas científicas com este propósito, pois há necessidade de encontrar uma alternativa que possa contribuir para a aderência da mãe ao seguimento ambulatorial, visto que uma simples avaliação da mamada pode identificar possíveis dificuldades relacionadas à pega do bebê e à lactação e nesta situação cabe ao fonoaudiólogo orientar adequadamente esta mãe com o intuito de favorecer o aleitamento materno.

Finalmente, destaca-se a importância da intervenção fonoaudiológica, especialmente na estimulação da sucção não-nutritiva e da estimulação oral em prematuros e recémnascidos e baixo peso ao nascer, uma vez que tais intervenções influenciam de forma positiva o aleitamento materno na alta hospitalar e no sexto mês de vida( ${ }^{(26,27)}$.

Aponta-se como uma das limitações do presente estudo o reduzido número de sujeitos no seguimento ambulatorial. Novas pesquisas são necessárias para subsidiar o direcionamento das ações fonoaudiológicas junto a essa população de risco.

\section{CONCLUSÃO}

A prevalência do AM, tanto na alta hospitalar quanto no sexto mês de vida, fica bastante aquém da recomendação da Organização Mundial da Saúde e seu aumento deve ser prioridade na atuação do fonoaudiólogo em Unidade de Terapia Intensiva Neonatal e no seguimento após a alta hospitalar.

Verifica-se, com o estudo, a necessidade de reorganização dos serviços de saúde, tanto em nível ambulatorial quanto hospitalar, na assistência à saúde da mulher e da criança, favorecendo o aumento da prevalência do aleitamento materno exclusivo até o sexto mês de vida.

\begin{abstract}
Purpose: To verify the prevalence of breastfeeding in preterm infants weighting over $1500 \mathrm{~g}$ at hospital discharge and until six months of life. Methods: This is a quantitative descriptive observational retrospective study, based on the analysis of records of infants born between January 2006 and December 2007, which were assisted by the Speech-Language Pathology department in the pediatric unit and the Speech-Language Pathology outpatient clinic of a hospital. The inclusion criteria were: low birth weight preterm infants, with no congenital heart or pulmonary diseases, no genetic syndromes, and APGAR score higher than three at one minute and five at five minutes. Results: The results from the 156 records studied showed that the prevalence of exclusive breastfeeding at hospital discharge was $58.3 \%$. At six months it was observed a prevalence of $22.2 \%$ of exclusive breastfeeding. Conclusion: The prevalence of exclusive breastfeeding was well below the World Health Organization recommendation, which is exclusive breastfeeding until the sixth month of life.
\end{abstract}

Keywords: Infant, newborn; Birth weight; Premature; Breast feeding; Weaning

\title{
REFERÊNCIAS
}

1. Nascimento MBR, Issler H. Aleitamento materno em prematuros: manejo clínico hospitalar. J Pediatr (Rio J). 2004;80(5 Supl):S163-72.

2. Brasil. Ministério da Saúde. Secretaria de Políticas de Saúde. Atenção humanizada ao recém-nascido de baixo peso, método mãe-canguru: manual do curso. Brasília: Ministério da Saúde; 2002.

3. Schanler RJ, Hurst NM, Lau C. The use of human milk and breastfeeding in premature infants. Clin Perinatol. 1999;26(2):379-98, vii.

4. Hall RT. Nutritional follow-up of the breastfeeding premature infant after hospital discharge. Pediatr Clin North Am. 2001;48(2):45360. Review.

5. Hylander MA, Strobino DM, Dhanireddy R. Human milk feedings and infection among very low birth weight infants. Pediatrics.
1998;102(3):E38.

6. Hylander MA, Strobino DM, Pezzullo JC, Dhanireddy R. Association of human milk feedings with a reduction in retinopathy of prematurity among very low birthweight infants. J Perinatol. 2001;21(6):35662. Comment in: J Perinatol. 2001;21(6):349.

7. Boo NY, Puah CH, Lye MS. The role of expressed breastmilk and continuous positive airway pressure as predictors of survival in extremely low birthweight infants. J Trop Pediatr. 2000;46(1):15-20.

8. Sanches MTC. Manejo clínico das disfunções orais na amamentação. J Pediatr (Rio J). 2004;80(5 Supl):S155-62.

9. Aguayo J. Maternal lactation for preterm newborn infants. Early Hum Dev. 2001;65(Suppl 2):S19-29.

10. Serra SOA, Scochi CGS. Dificuldades maternas no processo de 
aleitamento materno de prematuros em uma UTI neonatal. Rev Latinoam Enferm. 2004;12(4):597-605.

11. Pereira MG. Epidemiologia: teoria e prática. Rio de Janeiro: Guanabara Koogan; 1995.

12. Bicalho-Mancini PG, Velásquez-Meléndez G. Aleitamento materno exclusivo na alta de recém-nascidos internados em berçário de alto risco e os fatores associados a essa prática. J Pediatr (Rio J). 2004;80(3):2418.

13. Aquino RR, Osório MM. Alimentação do recém-nascido pré-termo: métodos alternativos de transição da gavagem para o peito materno. Rev Bras Saúde Matern Infant. 2008;8(1):11-6.

14. Pedras CTPA, Pinto EALC, Mezzacappa MA. Uso do copo e da mamadeira e o aleitamento materno em recém-nascidos prematuros e a termo: uma revisão sistemática. Rev Bras Saúde Matern Infant. 2008;8(2):163-9.

15. de Aquino RR, Osório MM. Relactation, translactation and breastorogastric tube as transition methods in feeding preterm babies. J Hum Lact. 2009;25(4):420-6.

16. Scochi CGS, Ferreira FY, Góes FSN, Fujinaga CI, Ferecini GM, Leite AM. Alimentação láctea e prevalência do aleitamento materno em prematuros durante internação em um hospital amigo da criança de Ribeirão Preto-SP, Brasil. Ciênc Cuid Saúde. 2008;7(2):145-54.

17. Alves AML, Silva EHAA, Oliveira AC. Desmame precoce em prematuros participantes do Método Mãe Canguru. Rev Soc Bras Fonoaudiol. 2007;12(1):23-8.

18. Howard CR, de Blieck EA, ten Hoopen CB, Howard FM, Lanphear BP, Lawrence RA. Physiologic stability of newborns during cup- and bottlefeeding. Pediatrics. 1999;104(5 Pt 2):1204-7.

19. Foiadelli F, Buhler KEB, Oliveira FM. O aleitamento materno em recém-nascidos pré-termo um mês após a alta hospitalar. Fono Atual. 2005;8(33):41-53.

20. Vituri SC, Brito ASJ. Prevalência do aleitamento materno em crianças até o sexto mês de idade na cidade de Maringá, estado do Paraná, Brasil. Acta Sci Health Sci. 2003;25(2):141-6.

21. Pedras CTPA. Copo ou mamadeira: evolução da transição alimentar, frequência e duração do aleitamento materno em recém-nascidos prematuros [dissertação]. Campinas: Faculdade de Ciências Médicas da Universidade Estadual de Campinas; 2007.

22. Parizotto J, Zorzi NT. Aleitamento Materno: fatores que levam ao desmame precoce no município de Passo Fundo, RS. Mundo Saúde (1995). 2008;32(4):466-74.

23. Ramos VW, Ramos JW. Aleitamento materno, desmame e fatores associados CERES. 2007;2(1):43-50.

24. Del Ciampo LA, Daneluzzi JC, Ricco RG, Martinelli Junior CE, Ferraz IS. Aleitamento materno exclusivo: do discurso à prática. Pediatria (São Paulo). 2008;30(1):22-6.

25. Escobar AMU, Ogawa AR, Hiratsuka M, Kawashita MY, Teruya PY, Grisi S, Tomikawa SO. Aleitamento materno e condições sócioeconômico-culturais: fatores que levam ao desmame precoce. Rev Bras Saúde Matern Infant. 2002;2(3):253-61.

26. Pimenta HP, Moreira MEL, Rocha AD, Gomes Junior SC, Pinto LW, Lucena SL. Efeitos da sucção não-nutritiva e da estimulação oral nas taxas de amamentação em recém-nascidos pré-termo de muito baixo peso ao nascer: um ensaio clínico randomizado. J Pediatr (Rio J). 2008;84(5):423-7.

27. Rocha MS, Delgado SE. Intervenção fonoaudiológica em recém-nascido pré-termo com gastrosquise. Rev Soc Bras Fonoaudiol. 2007;12(1):5562. 